\title{
Low earth orbit sounder retrieval products at geostationary earth orbit spatial and temporal scales
}

\author{
James Anheuser, ${ }^{\text {a,* }}$ Elisabeth Weisz, ${ }^{\text {b }}$ and W. Paul Menzel $\odot^{b}$ \\ ${ }^{a}$ University of Wisconsin-Madison, Atmospheric Oceanic and Space Sciences, Madison, \\ Wisconsin, United States \\ ${ }^{b}$ University of Wisconsin-Madison, Space Science and Engineering Center, Madison, \\ Wisconsin, United States
}

\begin{abstract}
Infrared (IR) sounders, onboard satellites in low Earth orbit, are high spectral resolution interferometers capable of providing very accurate IR radiance measurements using thousands of channels. Though their temporal resolution is a relatively low $12 \mathrm{~h}$, these data can be used to produce high vertical resolution temperature and humidity profiles. Imagers, often aboard satellites in geostationary Earth orbit, are radiometers providing high spatial and temporal resolution radiance measurements across a more limited number of bands, typically $\sim 10$ to $\sim 20$. Their data are used for monitoring Earth's weather and climate. A data fusion method (developed and previously demonstrated at University of Wisconsin-Madison) is utilized to construct high vertical resolution, sounder-like temperature and humidity retrievals at imager high spatial and temporal resolution. Using the Crosstrack Infrared Sounder (CrIS) and Advanced Baseline Imager (ABI) data, comparison of concurrent and $~ 50$ - min time-series fusion to radiosondes launched from February 2017 to March 2019 over the Department of Energy Atmospheric Radiation Measurement Southern Great Plains Site shows that the improved spatial and temporal resolution does not come at the expense of accuracy as compared with the sounder retrieval at native resolution. (c) The Authors. Published by SPIE under a Creative Commons Attribution 4.0 Unported License. Distribution or reproduction of this work in whole or in part requires full attribution of the original publication, including its DOI. [DOI: 10.1117/1.JRS.14.048502]
\end{abstract}

Keywords: infrared sounders; infrared imagers; data fusion.

Paper 200518 received Jul. 7, 2020; accepted for publication Oct. 9, 2020; published online Oct. 23, 2020.

\section{Introduction}

Data fusion is the process by which synergy is identified and utilized between two unique data sources. ${ }^{1}$ In remote sensing, data fusion has been used to construct infrared (IR) imager channels from IR sounder observations to maintain continuity, ${ }^{2}$ to construct true color imagery when the green color channel is not available, ${ }^{3}$ and to produce high spatial and temporal resolution surface reflectance products. ${ }^{4}$

The synergy utilized here is the complementing vertical, horizontal, and temporal resolutions of IR imagers and the high spectral resolution (or hyperspectral) sounders (Table 1). IR sounder/ imager fusion has been shown to be capable of constructing radiances with sounder-like high spectral resolution at geostationary imager-like high temporal and horizontal resolution. ${ }^{5}$ Measured radiances with high spectral resolution enable high vertical resolution in retrieved temperature and moisture profiles. When the fusion technique is applied directly to the hyperspectral sounder retrievals rather than radiances, sounder/imager fusion allows for construction of the aforementioned high vertical resolution, polar-orbiting sounder-like retrievals at geostationary imager temporal and horizontal resolution. ${ }^{6}$ These fusion products have the vertical resolution of an IR sounder $(\sim 1 \mathrm{~km})$ and the horizontal and temporal resolutions of an IR imager ( 2 to $4 \mathrm{~km}$ and as high as $1 \mathrm{~min}$, respectively). As such, sounder/imager fusion offers improvements over both sounder and imager data alone.

Sounder/imager fusion as applied to radiances has been validated against legacy channels available on Moderate Resolution Imaging Spectroradiometer, ${ }^{5}$ leading to the supposition that

*Address all correspondence to James Anheuser, anheuser@wisc.edu 
Table 1 Summary of CrIS and $\mathrm{ABI}$ attributes. Adapted from Ref. 6. CrIS has a much higher number of channels than $\mathrm{ABI}$, while $\mathrm{ABI}$ has a much smaller FOV size at nadir.

\begin{tabular}{|c|c|c|c|c|c|c|}
\hline Instrument & Satellite & $\begin{array}{l}\text { IR sepctral } \\
\text { range }(\mu \mathrm{m})\end{array}$ & $\begin{array}{l}\text { No. of IR } \\
\text { channels }\end{array}$ & $\begin{array}{l}\text { FOV size } \\
\text { (nadir) }\end{array}$ & $\begin{array}{l}\text { Swath } \\
\text { width }\end{array}$ & Scanning \\
\hline CrIS & S-NPP, NOAA-20 & 3.9 to 15.4 & $\begin{array}{l}1305 \text { (NSR) } \\
2211 \text { (FSR) }\end{array}$ & $14 \mathrm{~km}$ & $2200 \mathrm{~km}$ & $\pm 48.6^{\circ}$ \\
\hline $\mathrm{ABI}$ & GOES-16, GOES-17 & 3.9 to 13.3 & 10 & $2 \mathrm{~km}$ & Full disk & \\
\hline
\end{tabular}

Note: NSR: normal spectral resolution; FSR: full spectral resolution

sounder/imager fusion as applied to retrievals may contain accurate and useful information. This product fusion has been demonstrated with sounder/imager pairs on the same polar-orbiting platform and with polar-orbiting sounders paired with geostationary imagers. ${ }^{6}$ The latter pairing offers the opportunity to add temporal fusion to spatial fusion. In this work, we provide statistical validation of sounder/imager retrieval product spatial and temporal fusion results using radiosonde data provided by the Department of Energy (DOE) Atmospheric Radiation Measurement (ARM) facility ${ }^{7}$ and model data from the National Oceanic and Atmospheric Association (NOAA) National Centers for Environmental Prediction (NCEP) Rapid Refresh (RAP) model. ${ }^{8}$

\section{Instruments}

A typical high spectral resolution (or hyperspectral) IR sounder measures the radiance emitted from the Earth system with thousands of very narrow and well-calibrated channels; e.g., the Atmospheric Infrared Sounder (AIRS), ${ }^{9}$ Infrared Atmospheric Sounding Interferometer, ${ }^{10}$ and Crosstrack Infrared Sounder (CrIS). ${ }^{11}$ An example of a high spectral resolution brightness temperature spectrum is shown in Fig. 1. The fine spectral resolution allows for the retrieval of accurate and detailed vertical temperature and moisture profiles, trace gas concentrations, and cloud and surface parameters. ${ }^{9,12-14}$ Broad-band sounders and imagers, on the other hand, are not capable of capturing this level of detail in vertical structure because their sensitivity to spectral differences in IR radiation is much lower, as illustrated by the example of the broad spectral response functions (SRFs) in Fig. 1. In this work, high spectral resolution retrieval products are derived using the University of Wisconsin Dual Regression (UW DR) retrieval algorithm. ${ }^{15,16}$ Other retrieval algorithms proficient in processing high spectral resolution measurements can be utilized as well, e.g., the operational NOAA Unique Combined Atmospheric Processing System (NUCAPS). ${ }^{17}$ There are many types of high spectral resolution retrieval methodologies. Whereas NUCAPS includes microwave and IR data and an optimal estimation step, which improves the retrieval yield and sounding accuracy under certain conditions, the UW DR algorithm is based on linear regression with non-linearities addressed through classified statistics and various decision steps. In addition, the UW DR algorithm provides atmospheric profiles and surface and



Fig. 1 Example CrIS observed brightness temperature spectrum (blue, left axis) and ABI IR channel SRFs (red, right axis) plotted versus wavenumber. 
cloud parameters under clear and cloudy sky conditions at single field of view (FOV) horizontal resolution. The main differences between these two hyperspectral retrieval systems are described in Ref. 18. In general, hyperspectral sounder algorithms are capable of retrieving vertical temperature and humidity profiles with an accuracy of $1 \mathrm{~K}$ and $20 \%$ humidity, respectively, within a $1-\mathrm{km}$ layer. ${ }^{17,19,20}$ The information from these high spectral radiance measurements and the associated accurate vertical profiles are beneficial to forecasting, ${ }^{12,21}$ model assimilation, ${ }^{22,23}$ and research applications. ${ }^{13}$

Many sounders, such as CrIS aboard Suomi-National Polar-orbiting Partnership (S-NPP) and the Joint Polar Satellite System (JPSS) series, are in polar orbit. ${ }^{11}$ This means that, while their radiance data are available for the entire globe, most notably in regions where in-situ observations are sparse, its $\sim 12 \mathrm{~h}$ temporal resolution is low (Table 1). Additionally, sounders have a large FOV size, typically $\sim 14 \mathrm{~km}$. This limits the ability of sounders to provide the type of time evolution or high horizontal resolution information often needed in nowcasting settings.

IR imagers have a much lower number of channels compared with sounders. These channels are used to provide information about the earth surface and atmosphere. A typical imager has roughly 10-IR channels, with each channel often corresponding to a particular characteristic such as mid-level water vapor or ozone. ${ }^{24}$ The low spectral resolution, however, limits imagers ability to create high vertical resolution profiles of atmospheric characteristics. This low spectral resolution is illustrated by the SRFs of 10 broad IR bands plotted in Fig. 1.

In contrast to spectral or vertical resolution, imagers typically have very high horizontal resolution (Table 1) - sometimes on the order of hundreds of meters. ${ }^{25,26}$ This allows for the depiction of spatially small features in the atmosphere such as overshooting tops in a developing convective system. ${ }^{27}$ Imagers, such as the Advanced Baseline Imager (ABI), are often aboard geostationary satellites. While these platforms are limited to a single viewing angle and cannot view the high latitudes, they are able to provide much higher temporal resolution, sometimes as high as 1 min. ${ }^{24}$ This makes imagers onboard geostationary platforms ideal for providing the high temporal and spatial resolution information used in nowcasting settings, albeit without highly resolved vertical information.

\section{Fusion Process Methods and Results}

The fusion process described herein has been utilized to construct both radiances ${ }^{5}$ and retrievals. ${ }^{6}$ As described in Refs. 5 and 6, a multi-dimensional (k-d) tree search algorithm applied to imager radiances and geolocation is used to find a number $(N)$ of best matching sounder FOVs for each imager pixel. The sounder and imager data need not be concurrent, though near concurrent is favored. In radiance fusion, the radiances of these $N$ closest matching FOVs are averaged for each pixel to construct fusion radiances. In retrieval product fusion, as utilized here, the retrievals corresponding to the $N$ closest matching sounder FOVs are averaged at every pressure level to construct retrievals corresponding to each imager pixel. The resulting fusion retrievals show the characteristics of the atmosphere at the vertical resolution of the sounder and at the horizontal resolution and observation time of the imager data. All plots and illustrations shown in this section were created using CrIS granules starting at 1748 UTC and 1754 UTC on 19 May 2017 over the Chesapeake Bay, Virginia, region and the Geostationary Operational Environmental Satellite (GOES)-16 ABI images taken from 1800 UTC to 2100 UTC on the same day.

\subsection{Retrieval Product Fusion}

In both radiance fusion and retrieval product fusion, a k-d tree search algorithm is utilized to find matches among the vectors of a training dataset to vectors within a query dataset. The k-d tree is a nearest neighbor search that is computationally fast and efficient and can be applied to spaces of arbitrary dimensionality. ${ }^{28}$

Imager pixel geolocation and radiance data form the query dataset. The imager pixels are geographically collocated to the sounder FOVs and the radiances are averaged within each FOV to form the training dataset. In other words, high and low spatial resolution imager data form the query and training datasets, respectively. The k-d tree search is then used to match each imager 
pixel from the query dataset with a set of $N$ best matching sounder FOVs represented by the low spatial resolution imager data in the training dataset. This first step is identical between both radiance and product fusion, and in both cases, hyperspectral sounder data (radiance or retrievals) are not yet used. A cloud mask can be applied to the imager data prior to this process; this saves computing time by eliminating pixels from the query dataset that are known to be cloudy. In this case study, a cloud mask has been applied to the ABI data. ${ }^{29}$

In the second step, the sounder data from the $N$ best matching FOVs for each pixel are averaged. Here, in retrieval product fusion, it is the sounder retrievals that are averaged. A minimum number of non-cloudy neighbors out of $N$ for each pixel at a given pressure level is set, below which the pixel is assigned as cloudy at the pressure level in question. The result is a high vertical resolution retrieval profile for every clear sky imager pixel. These new data retain the high vertical resolution of the sounder derived retrieval along with the high horizontal resolution of the imager.

The combination of parameters taken to be optimized for this application is a best match subset of $N=5$, a minimum clear best match requirement of minimum $N_{\mathrm{clr}}=2$ and the ABI input channel combination of $8,9,10,11,13,14,15$, and 16 (corresponding to wavelengths at $6.2,7,7.3,8.5,10.3,11.2,12.3$, and $13.3 \mu \mathrm{m}){ }^{24}$ In this section, a summary of the resultant Chesapeake Bay, Virginia, 19 May 2017 at 1800 UTC retrieval product fusion dataset is presented in comparison with the original ABI and CrIS data from the same time. The data here illustrates the synergy of the imager's high horizontal resolution and the sounder's high vertical resolution.

The imager inputs into the fusion process are shown in Fig. 2. The high horizontal resolution of the imager data is immediately evident. The horizontal resolution is $2 \mathrm{~km}$ at nadir and 3 to $4 \mathrm{~km}$ over the region shown due to the higher viewing zenith angle. The goal is to retain this high horizontal resolution in the final result. While these image inputs do not contain highly resolved vertical information, each channel does offer information content with respect to a particular atmospheric characteristic on which the k-d tree search process can train. For instance, inclusion of ABI channel 9, known as the mid-level water vapor channel, ${ }^{24}$ allows the $\mathrm{k}-\mathrm{d}$ tree search to be guided by mid-level water vapor when matching imager pixels with sounder FOVs. The sounder inputs (Fig. 3) contain highly resolved vertical information about the atmosphere but do not have the high horizontal resolution of the imager. The CrIS products used here were retrieved using the full spectral resolution (FSR) data ${ }^{19}$ (2211 channels). Other combinations of imager/sounder instrument pairs and retrieval methods capable of processing hyperspectral sounder radiances may be used in a similar fusion framework described here, but they are not presented herein. As discussed throughout this work, the intent of this fusion process is to capture the high horizontal resolution of the imager and the high vertical resolution of the sounder.

Retrieval product fusion using the $N=5$, minimum clear $N_{\text {clr }}=2$, and eight-channel search are shown in Fig. 4. The data do exhibit the high horizontal resolution of the ABI imager as

(a)

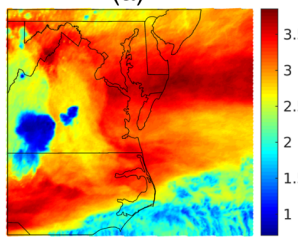

(e)

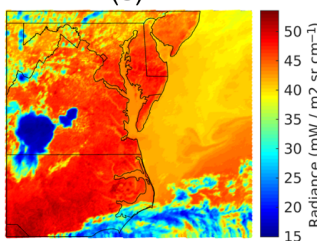

(b)

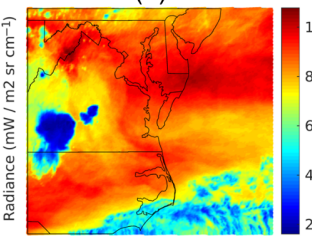

(f)

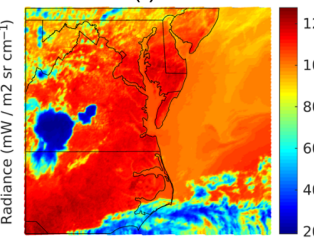

(c)

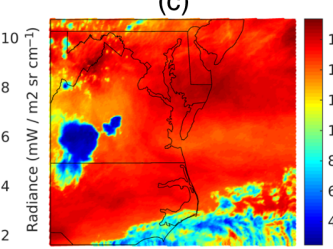

(g)

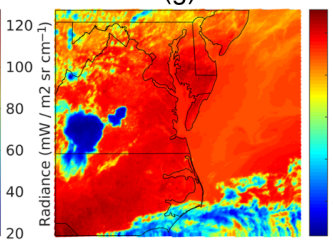

(d)

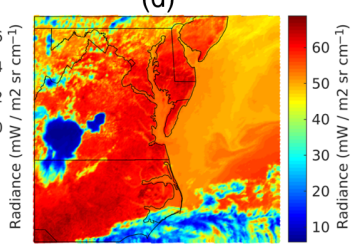

(h)

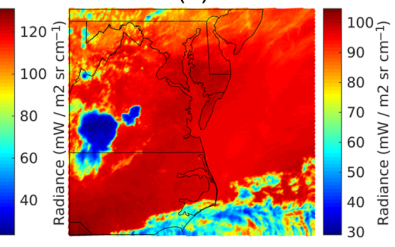

Fig. 2 ABI brightness temperatures from channels (a) 8; (b) 9; (c) 10; (d) 11; (e) 13; (f) 14; (g) 15; and (h) 16, taken aboard GOES-16 over the Chesapeake Bay region at 1800 UTC on 19 May 2017. These images serve as the imager input into the fusion process example shown here. 
(a)

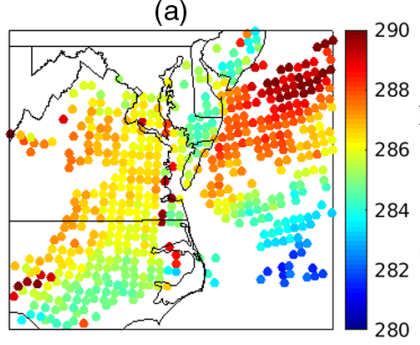

(d)

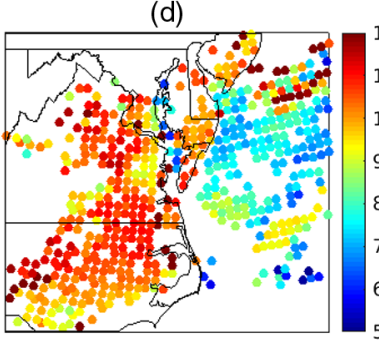

(b)

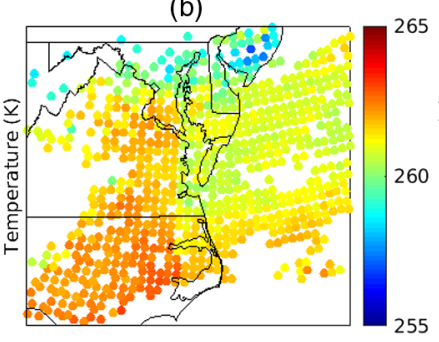

(e)

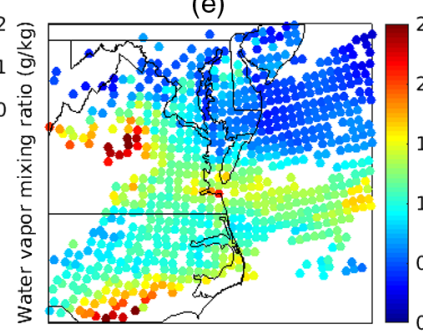

(c)

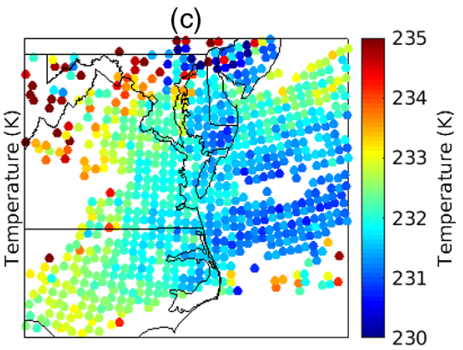

(f)

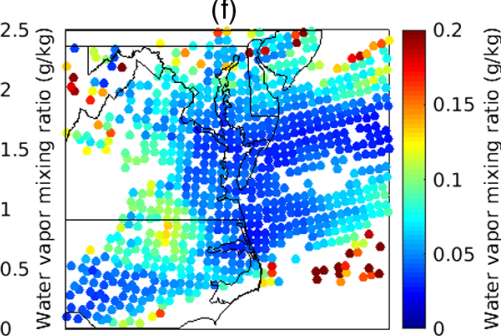

Fig. 3 CrIS derived temperature retrievals at (a) $850 \mathrm{hPa}$; (b) $500 \mathrm{hPa}$; and (c) $300 \mathrm{hPa}$ and water vapor mixing ratio retrievals at (d) $850 \mathrm{hPa}$; (e) $500 \mathrm{hPa}$; and (f) $300 \mathrm{hPa}$, over the Chesapeake Bay region at 1754 UTC on 19 May 2017 created using the UW DR retrieval algorithm. ${ }^{15,16}$ These data serve as the sounder input into the fusion process example shown here.

(a)

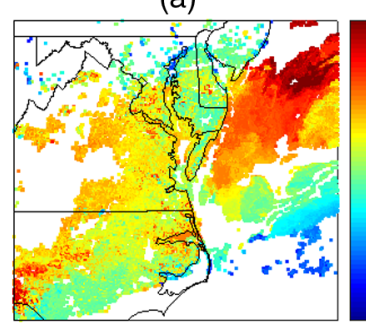

(d)

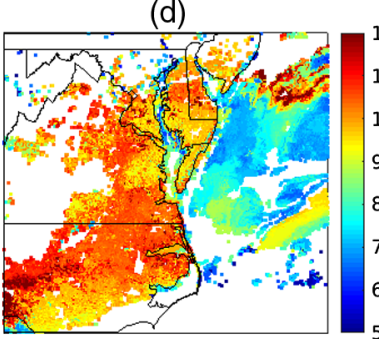

(b)

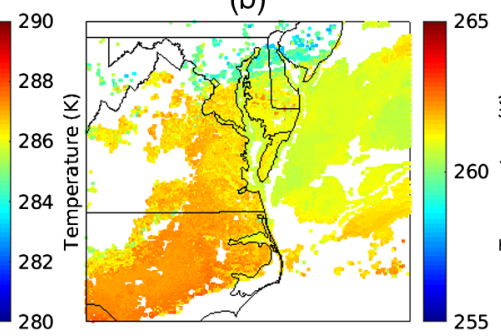

(e)

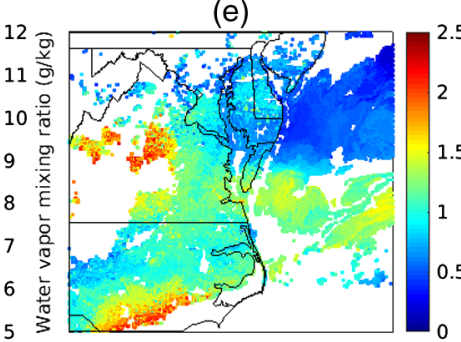

(c)



(f)

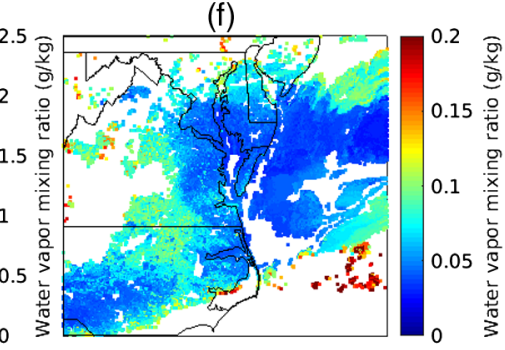

Fig. 4 Retrieval product fusion temperature retrievals at (a) $850 \mathrm{hPa}$, (b) $500 \mathrm{hPa}$, and (c) $300 \mathrm{hPa}$ and water vapor mixing ratio retrievals at (d) $850 \mathrm{hPa}$, (e) $500 \mathrm{hPa}$, and (f) $300 \mathrm{hPa}$, over the Chesapeake Bay region at 1754 UTC on 19 May 2017 created using ABI and the UW DR retrieval algorithm $^{15,16}$ applied to CrIS radiances.

intended. Additionally, the products are "sounder-like" in that they have vertical information of temperature and water vapor. In other words, the spatial fusion of ABI/CrIS increases the horizontal resolution of the soundings by roughly an order of magnitude. This work assesses the accuracy of this retrieval product fusion.

\subsection{Retrieval Product Fusion Time Series}

The high temporal resolution of the ABI imager allows for the creation of time series of retrieval product fusion data. GOES ABI images are available at a refresh rate of 1 min inside one of two mesoscale regions over the contiguous United States (CONUS), 5 min over CONUS, and 15 min 
over the entire disk. ${ }^{24}$ This temporal fusion begins at "time step 0 " with retrieval product fusion as described in Sec. 3.1 between sounder data and imager data that are very near concurrent in time, i.e., the ABI data is that which is closest in time to the CrIS overpass. "Time step 1" occurs when the next ABI image is available for the region in question. At time step 1, a new retrieval product fusion is created as follows. The $\mathrm{k}-\mathrm{d}$ tree is used to match each imager pixel at time step 1 with the $N$ best matching imager pixels from time step 0 . This means that every pixel in the new imager data from time step 1 is matched with a subset of the best match (in radiance and geolocation) imager pixels from time step 0 . The corresponding $N$ fusion retrievals from time step 0 (already at high spatial resolution) are then averaged to form the new fusion retrieval for that pixel at time step 1. This process can be repeated as new ABI data continuously becomes available at subsequent time steps with each new imager pixel being matched with $N$ pixels from the previous image, the high spatial resolution retrievals of which are then averaged to form the retrieval product fusion at the new time step. As before, the best match subset is taken to be of size $N=5$, and the minimum number of clear neighbors is taken to be minimum clear $N_{\text {clr }}=2$.

Figures 5 and 6 show an example time series created by utilizing the spatial/temporal fusion approach, i.e., completing the time step 0 spatial fusion at 1800 UTC and using the temporal fusion procedure to create retrieval product fusion at 2000 UTC and 2200 UTC. The retrieval product fusion constructs a more coherent depiction of the atmospheric changes at the overpass time and over the following $4 \mathrm{~h}$. A detailed description of the atmospheric processes observed in the retrieval product fusion time series of this case study, including a comparison with ABI and NCEP RAP model data, can be found in Sec. 3.3 of Ref. 6. After the 4-h period shown in Fig. 5, the time series no longer tracks the convective development cycle very well. More work is necessary to determine for how long after the sounder overpass temporal fusion remains useful.

(a)

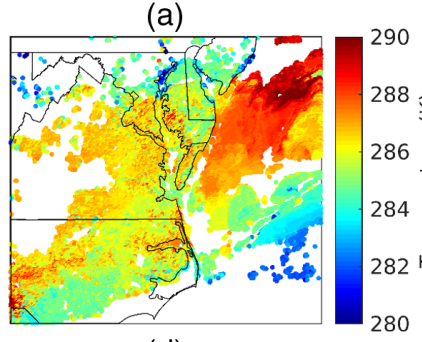

(d)

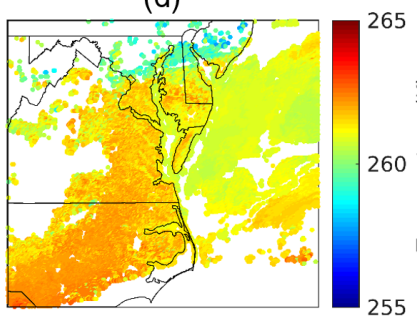

(g)

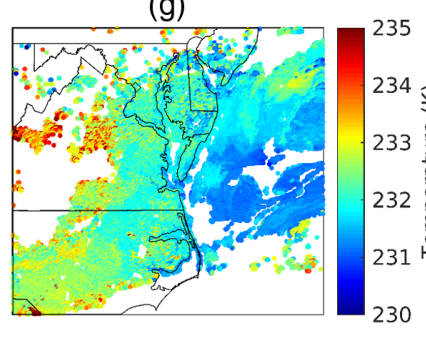

(b)

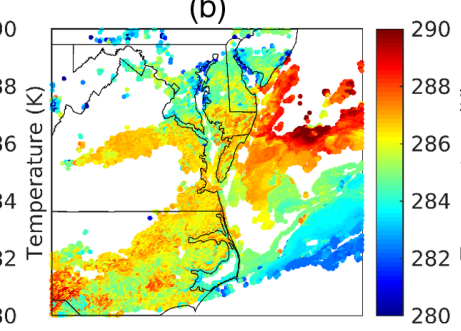

(e)

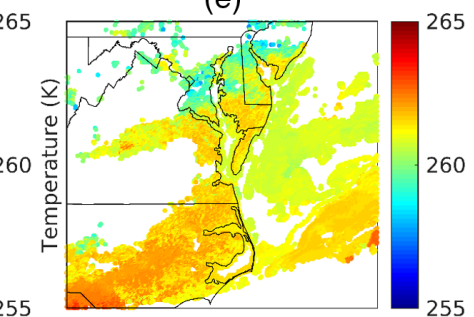

(h)

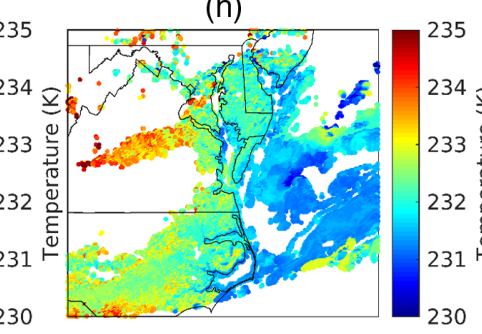

(c)

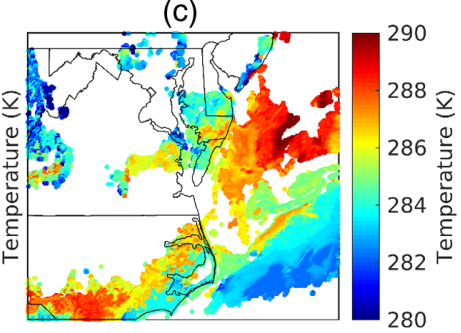

(f)

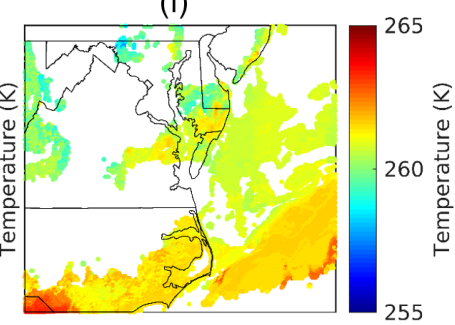

(i)

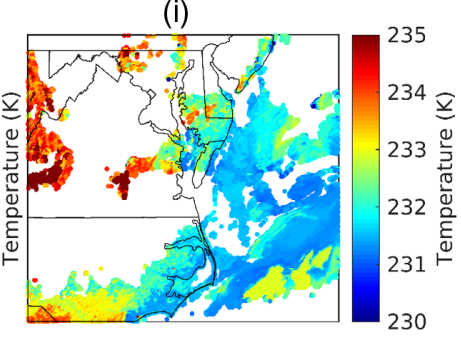

Fig. 5 Fusion temperature and at (a)-(c) $850 \mathrm{hPa}$; (d)-(f) $500 \mathrm{hPa}$; and (g)-(i) $300 \mathrm{hPa}$ at (a), (d), and (g) 1800 UTC; (b), (e), and (h) 2000 UTC; and (c), (f), and (i) 2200 UTC over Chesapeake Bay using the ABI image from 1800 UTC and the CrIS data from the 1748 UTC and 1754 UTC granules on 19 May 2017. White signifies the presence of cloud. Features in the atmosphere, especially convective lift shown by the $500 \mathrm{hPa}$ temperature, can be followed over time. 
(a)

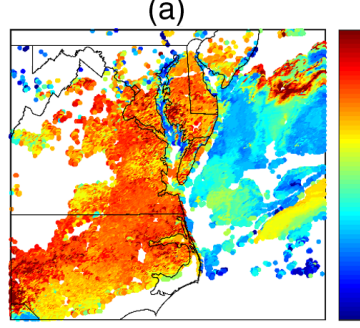

(d)

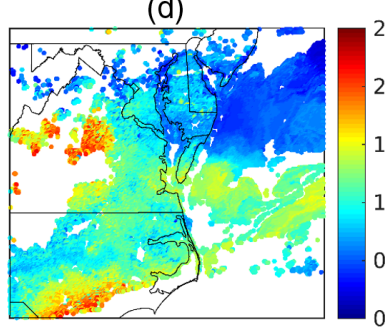

(g)

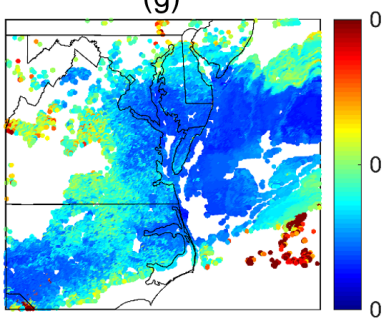

(b)

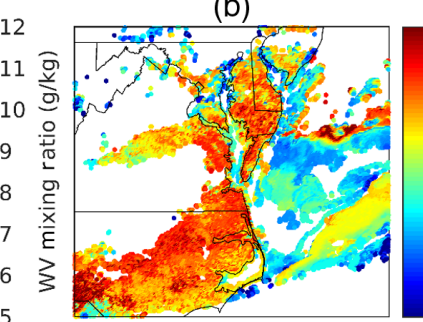

(e)

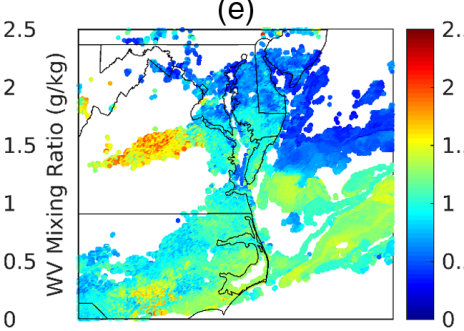

(h)

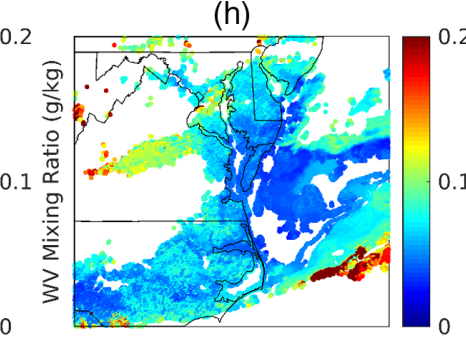

(c)

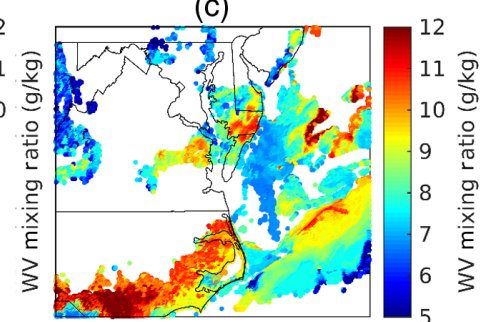

(f)



(i)

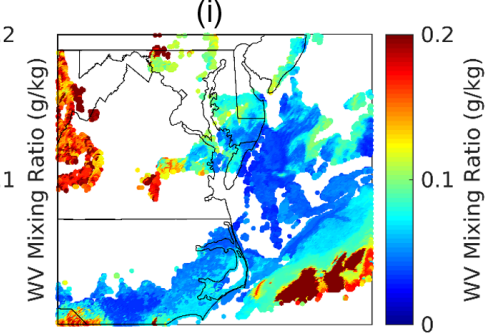

Fig. 6 Water vapor mixing ratio at (a)-(c) $850 \mathrm{hPa}$; (d)-(f) $500 \mathrm{hPa}$; and (g)-(i) $300 \mathrm{hPa}$ at (a), (d), and (g) 1800 UTC; (b), (e), and (h) 2000 UTC; and (c), (f), and (i) 2200 UTC over Chesapeake Bay using the ABI image from 1800 UTC and the CrIS data from the 1748 UTC and 1754 UTC granules on 19 May 2017. White signifies the presence of cloud. Features in the atmosphere, especially convective lift shown by the $500-\mathrm{hPa}$ water vapor mixing ratio, can be followed over time.

Overall, this case study offered interesting results and speaks to the potential for spatial and temporal product fusion in nowcasting settings. The near-real time retrieval system used operationally in this scenario is NUCAPS. Spatial/temporal product fusion using ABI radiance data can be applied to NUCAPS to enhance spatial resolution from $\sim 45 \mathrm{~km}$ to 2 to $4 \mathrm{~km}$ and temporal resolution from twice daily to every $15 \mathrm{~min}$ to further improve nowcasting and possibly forecasting operations.

\section{Validation}

Radiosondes are commonly used as ground truth reference for validating satellite data products. ${ }^{30,31}$ DOE ARM Southern Great Plains (SGP) radiosondes were launched to be concurrent with the operational CrIS overpasses ${ }^{32}$ initially S-NPP was designated as the operational spacecraft and later NOAA-20 (designated JPSS-1 prior to launch) was. These radiosonde data were used in this work to develop mean and standard deviation error profiles for CrIS/ABI product fusion. S-NPP precedes NOAA-20 by roughly $50 \mathrm{~min}$ (1/2 orbit); thus temporal fusion retrievals with time step 0 at S-NPP overpass time and final time step at NOAA-20 overpass time can also be validated. The RAP model and CrIS UW DR error statistic profiles are added to provide additional context.

\subsection{Radiosonde Dataset}

Though radiosonde data have often been used as validation for satellite derived profiles of the atmosphere, there are inherent limitations. To combat some of the potential error sources, best 
estimate radiosonde profiles were created coincident with S-NPP overpasses. Each best estimate profile is comprised of two radiosonde launches - one at roughly 45 min prior to satellite overpass and one at roughly 5 min prior to satellite overpass. The two radiosonde profiles are linearly interpolated to the overpass time at every pressure level to best represent the state of the atmosphere at the overpass time. The water vapor profile is scaled by a height-independent factor such that the profile's total column precipitable water matches the total column precipitable water measured at the overpass time by a ground-based, two-channel microwave radiometer. Both of these strategies are similar to those described in Ref. 31. Best estimate profiles were not available for NOAA-20 overpasses; thus the radiosonde launched 5 min prior to overpass time was used. The radiosonde data from March 2017 to March 2018 are from radiosondes released coincident with S-NPP overpasses, and the radiosonde data from March 2018 to April 2019 are from radiosondes released coincident with NOAA-20 overpasses.

\subsection{Retrieval Product Fusion Dataset}

To create a statistical comparison between retrieval product fusion and the radiosonde dataset, a retrieval product fusion profile was created for every radiosonde profile included in the dataset. The method by which the retrieval product fusion profile was created depends on whether the sounder overpass and fusion time are concurrent or the fusion is created via the time-series method described in Sec. 3.2.

For validation when the sounder, fusion, and radiosonde are concurrent, retrieval product fusion was completed using the CrIS granules from the overpass targeted by the radiosonde launch and the GOES-16 ABI image from nearest in time to the radiosonde launch. The CrIS granules always include the ARM SGP radiosonde launch site. Fusion was completed for the $\sim 30 \mathrm{ABI}$ pixels that are closest to the radiosonde launch site. The fusion profile from the pixel with the cloud-free profile reaching the lowest into the troposphere was chosen for validation. This fusion profile is then compared with the radiosonde to compute the statistical comparisons shown in Figs. 7 and 8.

For validation of fusion created via the time-series method described in Sec. 3.2, time step 0 was taken to be fusion between the CrIS granules from S-NPP and the GOES-16 ABI data from nearest in time to the S-NPP overpass. Time-series fusion was then created as described in
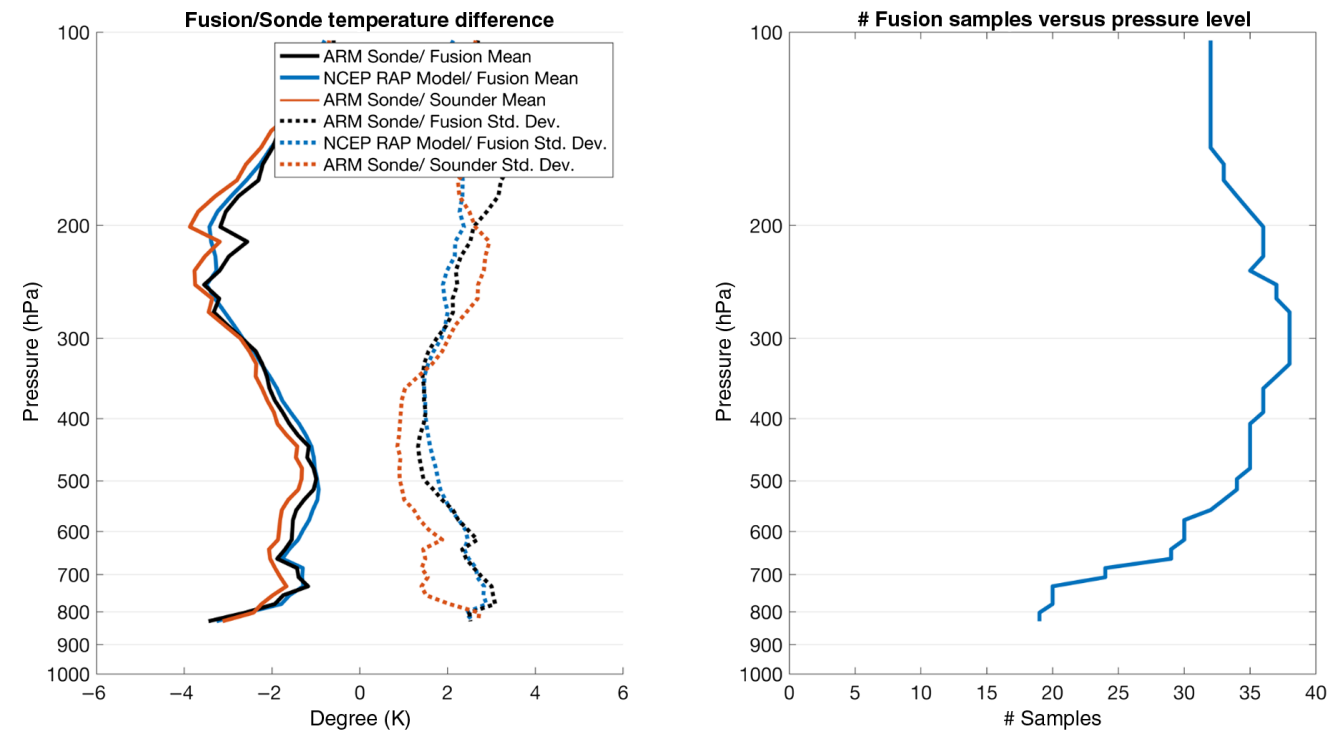

Fig. 7 (a) Mean (solid) and standard deviation (dashed) of temperature differences versus pressure level between sonde and fusion (black), model and fusion (blue) and sounder and sonde (orange) from February 2017 to February 2018 over the DOE ARM SGP site. (b) Number of clear samples versus pressure level for the period in question. Temperature bias in the mid troposphere is $\sim-1.5 \mathrm{~K}$ with a standard deviation of $\sim 2 \mathrm{~K}$. 

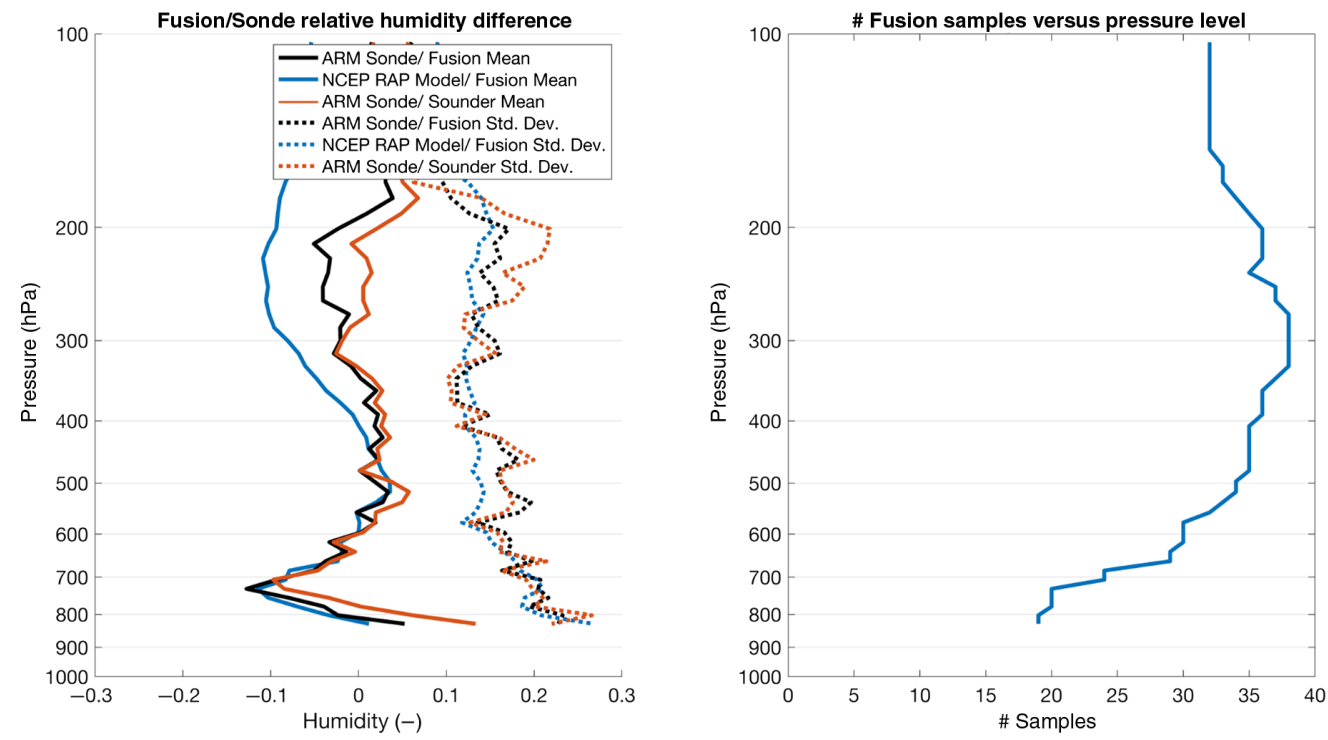

Fig. 8 (a) Mean (solid) and standard deviation (dashed) of relative humidity differences versus pressure level between sonde and fusion (blue) and model and fusion (orange) from February 2017 to February 2018 over the DOE ARM SGP Site. (b) Number of clear samples versus pressure level for the period in question. Relative humidity bias in the upper tropopause is $\sim 0$ with a $\sim-10 \%$ bias jump at $\sim 750 \mathrm{hPa}$. The standard deviation starts at $25 \%$ at the surface but decreases to $15 \%$ through the troposphere.
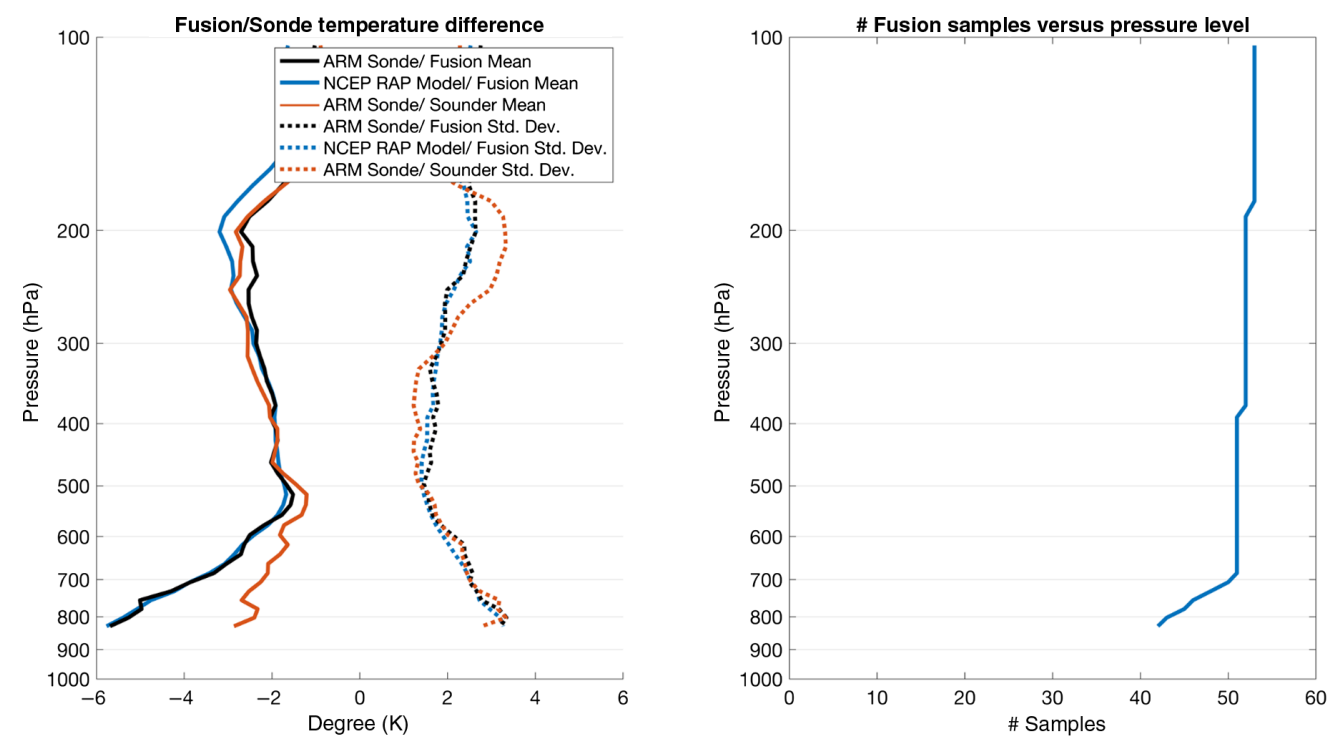

Fig. 9 (a) Mean (solid) and standard deviation (dashed) of temperature differences versus pressure level between sonde and time-series fusion using S-NPP CrIS (black), model and fusion (blue) and sounder and NOAA-20/JPSS-1 CrIS (orange) from February 2018 to March 2019 over the DOE ARM SGP site. (b) Number of clear samples versus pressure level for the period in question. Temperature bias in the mid- to upper-troposphere is $\sim-2 \mathrm{~K}$ to $\sim-3 \mathrm{~K}$ with a standard deviation of $\sim 1 \mathrm{~K}$ to $\sim 3 \mathrm{~K}$.

Sec. 3.2 forward in time to the ABI image closest to the corresponding NOAA-20 overpass. The NOAA-20 overpass is $\sim 50 \mathrm{~min}$ after the S-NPP overpass. The radiosonde launched $\sim 5 \mathrm{~min}$ before the NOAA-20 overpass was used for validation. The fusion profile at this later time is then compared with the radiosonde to compute statistical comparisons in Figs. 9 and 10 that represent validation of $\sim 50 \mathrm{~min}$ time-series fusion. 

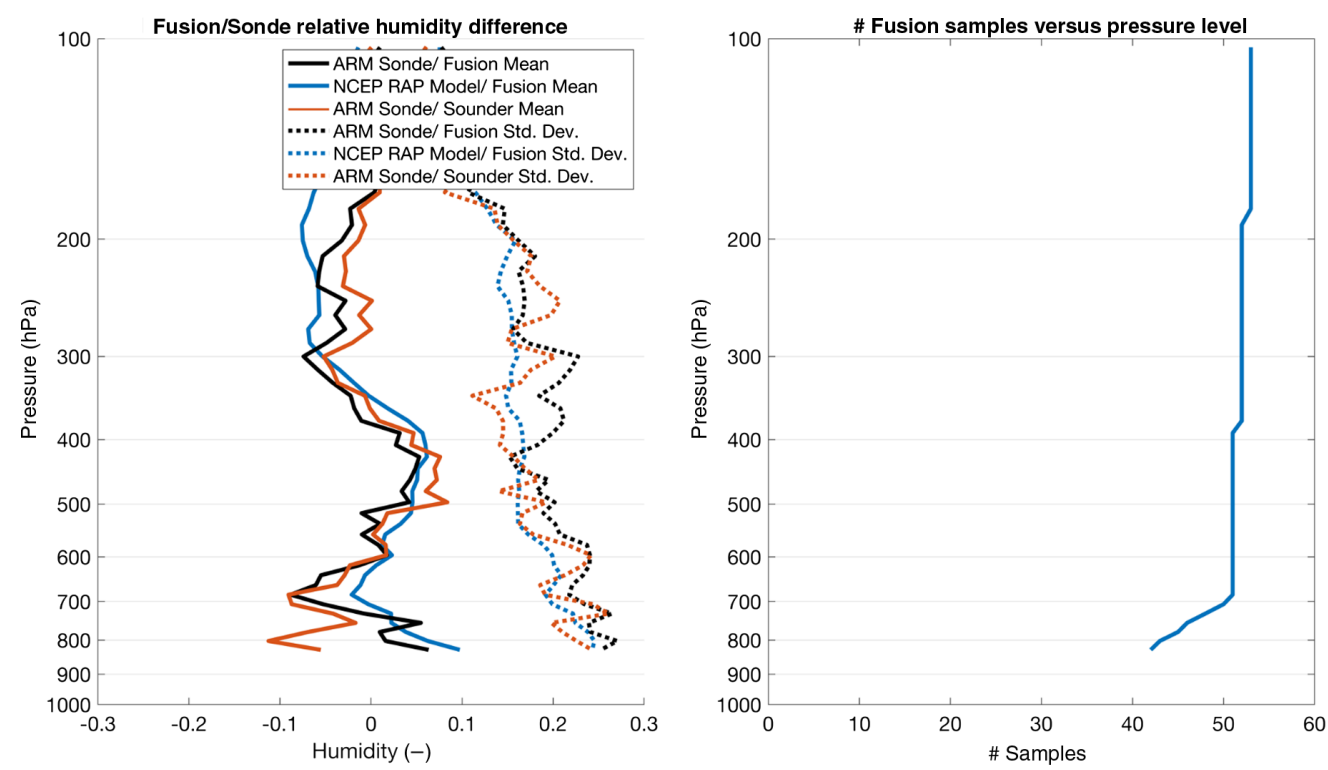

Fig. 10 (a) Mean (solid) and standard deviation (dashed) of relative humidity differences versus pressure level between sonde and time-series fusion using S-NPP CrIS (black), model and fusion (blue) and sounder and NOAA-20/JPSS-1 CrIS (orange) from February 2018 to March 2019 over the DOE ARM SGP site. (b) Number of clear samples versus pressure level for the period in question. Relative humidity bias in the tropopause oscillates between $10 \%$ and $-10 \%$ with a standard deviation decreasing from $25 \%$ at the surface to $20 \%$ through the upper troposphere. Microwave radiometer-based water vapor scaling was not available for these radiosonde samples.

\subsection{Validation Results}

\subsubsection{Spatial retrieval product fusion}

The spatial retrieval product fusion temperature created using concurrent CrIS and GOES-16 ABI compares favorably with the sondes and appears to perform equal to or better than the native resolution CrIS sounder retrievals (Fig. 7). Through much of the mid troposphere from near 750 to $400 \mathrm{hPa}$, the bias (mean) of the ARM sonde minus fusion temperature profile differences is between $-1 \mathrm{~K}$ and $-2 \mathrm{~K}$ with a standard deviation of $\sim 2 \mathrm{~K}$. Approaching the surface, the bias becomes more than $\sim-3 \mathrm{~K}$ at $850 \mathrm{hPa}$. Above $400 \mathrm{hPa}$, the bias is also $\sim-3 \mathrm{~K}$ and then heads toward $0 \mathrm{~K}$ approaching the tropopause. Throughout the profile, the fusion results appear to be as good as or better than the sounder alone.

The spatial retrieval product fusion relative humidity created using concurrent $\mathrm{CrIS}$ and GOES-16 ABI also compares favorably with the sondes and once again compares similarly with the results from the sounder alone (Fig. 8). Nearest to the surface, the bias is close to $0 \%$. Between about 850 and $650 \mathrm{hPa}$, the fusion, model, and sounder bias profiles move to $\sim-10 \%$ and then return to about $0 \%$. Above this point, the fusion and sounder profiles oscillate around $0 \%$ bias up to $350 \mathrm{hPa}$. The fusion bias increases once again above this point, but the best estimate water vapor profiles exhibit large uncertainties here $(10 \%){ }^{31}$

Throughout both the temperature and relative humidity difference profiles, spatial retrieval product fusion between concurrent CrIS and GOES-16 ABI data exhibits biases nearly identical to those of the native resolution CrIS retrieval. This result is more important than the bias data itself. Through the fusion process, the horizontal resolution of the CrIS retrievals has been greatly improved while their accuracy has remained unchanged. While the best estimate profiles have much improved uncertainties versus raw radiosonde data, much of the bias shown here may be due to measurement error. As such, these bias results can be taken to be upper bounds.

\subsubsection{Temporal retrieval product fusion}

The temperature difference profile for the CrIS and GOES-16 ABI data fusion carried forward $50 \mathrm{~min}$ through temporal retrieval product fusion (Fig. 9) exhibits similar behavior to the spatial 
retrieval product fusion except in the lower troposphere. At around $550 \mathrm{hPa}$, the bias is $\sim-2 \mathrm{~K}$. Moving up in the atmosphere, the bias goes to $\sim-3 \mathrm{~K}$ at $200 \mathrm{hPa}$, above which it begins to approach $0 \mathrm{~K}$. Below $550 \mathrm{hPa}$, the fusion and model bias of $\sim-2 \mathrm{~K}$ at $550 \mathrm{hPa}$ goes to nearly $\sim-6 \mathrm{~K}$ near the surface, while the sounder bias goes from $\sim-2 \mathrm{~K}$ at $550 \mathrm{hPa}$ to $\sim-3 \mathrm{~K}$ near the surface. It is unlikely that the temporal difference is causing this bias increase as a previous study has shown that a 60 min change in the atmosphere at the ARM SGP site shows $0 \mathrm{~K}$ bias. ${ }^{31}$ It is more likely that the absence of best estimate profiles for these cases has introduced uncertainty. The standard deviation of the temporal fusion temperature profile is nearly the same as that of the time coincident spatial fusion profile.

The temporal retrieval product fusion relative humidity compares favorably with the sondes (Fig. 10). Throughout the troposphere, the bias appears to oscillate between $-10 \%$ and $10 \%$ before approaching $0 \%$ in the upper troposphere. This oscillatory behavior is also exhibited by the sounder and model difference profiles. The variable nature of these biases may be due to the lack of water vapor scaling in the creation of best estimate profiles. The standard deviation of the temporal fusion relative humidity profile is similar to that of the time coincident spatial fusion profile except between 450 and $300 \mathrm{hPa}$ where it is $5 \%$ greater.

\section{Summary and Conclusion}

Independently, modern IR imagers and IR sounders aboard Earth-orbiting satellites exhibit unique advantages that make them useful and important tools to today's weather and climate monitoring operations. By identifying and utilizing an opportunity for synergy between the two types of instruments, the data fusion method demonstrated here produces results exhibiting the advantages of both while shedding some of their weaknesses. This fusion method has been previously proven to produce sounder-like, narrow band radiances at imager-like horizontal resolution to supplement existing imager bands. ${ }^{5}$ Here, we build upon a previous demonstration of spatial/temporal retrieval product fusion ${ }^{6}$ by further tuning this method to produce high vertical resolution retrieval products at high imager horizontal and temporal resolution. Validation is also presented through comparison of fusion retrievals to radiosonde profiles.

The fusion method utilizes a k-d tree search ${ }^{28}$ to find a subset of sounder FOVs that best matches each imager pixel in imager radiance and geolocation space. Hyperspectral sounder profile retrievals corresponding to the subset of FOVs are averaged at every pressure level to construct the fusion retrieval profile. In the case of $\mathrm{ABI} / \mathrm{CrIS}$ fusion, the resulting retrieval product has an ABI horizontal resolution of $\sim 2$ to $4 \mathrm{~km}$ while maintaining the CrIS high vertical resolution. Once this high spatial resolution data is available (at the CrIS measurement time), temporal fusion can be performed as new ABI images become available. In this process, the k-d tree search is applied to imager radiance data from consecutive time steps. This yields fusion retrieval results with high temporal resolution. By constructing a time series of hourly available fusion products, it is now possible to observe a storm system as it moves into pre-convective environments and watch in high resolution as convection moves warmer air aloft. These data in particular provide a glimpse of the utility that a geostationary hyperspectral sounder would provide to the meteorology community.

We utilized the radiosonde launches associated with the CrIS validation campaign at the DOE ARM SGP site ${ }^{7}$ to compare fusion results versus ground truth. Best estimate profiles similar to Ref. 31 and raw radiosonde profiles were used to study the most accurate quantifications of temperature and water vapor profiles possible. When compared with these best estimate profiles, fusion performs well. The nearest CrIS retrieval FOV was also compared with the radiosonde profile in each case and shows similar biases to the fusion results. This means that the fusion process improves the $\sim 14-\mathrm{km}$ horizontal resolution of the CrIS sounder retrieval to $\sim 2$ to $4 \mathrm{~km}$ with similar accuracy when compared with radiosondes.

Data fusion between instrument pairs, as demonstrated here, is a useful tool that can be applied to instrument groups exhibiting synergy to provide observations with characteristics not possible with individual satellites. Particularly interesting is the opportunity for data fusion among smallsats and cubesats as they become more prevalent. 


\section{Acknowledgments}

The authors gratefully acknowledge the support from NOAA under Grant No. NA15NES4320001. We acknowledge the use of best estimate radiosonde profiles through the NOAA NESDIS STAR JPSS Federal Funding Opportunity NOAA-NESDIS-NESDISPO2019-2005866 and the University of Wisconsin-Madison-based NOAA Cooperative Institute for Meteorological Satellite Studies (CIMSS) and NCEP RAP analysis data through the NOAA Operational Model Archive and Distribution System (NOMADS). CrIS and ABI level 1 data were provided courtesy of the Space Science and Engineering Center/University of WisconsinMadison. The authors declare no conflicts of interest.

\section{References}

1. L. Wald, "Some terms of reference in data fusion," IEEE Trans. Geosci. Remote Sens. 37(3), 1190-1193 (1999).

2. J. Cross et al., "Statistical estimation of a $13.3 \mu \mathrm{m}$ Visible Infrared Imaging Radiometer Suite channel using multisensor data fusion," J. Appl. Remote Sens. 7(1), 073473 (2013).

3. S. D. Miller et al., "A case for natural colour imagery from geostationary satellites, and an approximation for the GOES-R ABI," Int. J. Remote Sens. 33(13), 3999-4028 (2012).

4. F. Gao et al., "On the blending of the Landsat and MODIS surface reflectance: predicting daily Landsat surface reflectance," IEEE Trans. Geosci. Remote Sens. 44(8), 2207-2218 (2006).

5. E. Weisz, B. A. Baum, and W. P. Menzel, "Fusion of satellite-based imager and sounder data to construct supplementary high spatial resolution narrowband IR radiances," J. Appl. Remote Sens. 11(3), 036022 (2017).

6. E. Weisz and W. P. Menzel, "Imager and sounder data fusion to generate sounder retrieval products at an improved spatial and temporal resolution," J. Appl. Remote Sens. 13(3), 034506 (2019).

7. G. M. Stokes and S. E. Schwartz, "The atmospheric radiation-measurement (ARM) program-programmatic background and design of the cloud and radiation test-bed," Bull. Am. Meteorol. Soc. 75(7), 1201-1221 (1994).

8. S. G. Benjamin et al., "A North American hourly assimilation and model forecast cycle: the Rapid Refresh," Mon. Weather Rev. 144(4), 1669-1694 (2016).

9. H. H. Aumann et al., "AIRS/AMSU/HSB on the aqua mission: design, science objectives, data products, and processing systems," IEEE Trans. Geosci. Remote Sens. 41(2), 253-264 (2003).

10. P. Hebert et al., "IASI instrument: technical description and measured performances," Proc. SPIE 10568, 1056806 (2004).

11. Y. Han et al., "Suomi NPP CrIS measurements, sensor data record algorithm, calibration and validation activities, and record data quality," J. Geophys. Res. Atmos. 118(22), 12,73412,748 (2013).

12. S. A. Ackerman et al., "Satellites see the world's atmosphere," Meteor. Monogr. 59, 4.1-4.53 (2018).

13. M. T. Chahine et al., "Improving weather forecasting and providing new data on greenhouse gases," Bull. Am. Meteorol. Soc. 87(7), 911-926 (2006).

14. W. L. Smith et al., "Remote-sensing cloud properties from high-spectral-resolution infrared observations," J. Atmos. Sci. 50(12), 1708-1720 (1993).

15. W. L. Smith et al., "Dual-regression retrieval algorithm for real-time processing of satellite ultraspectral radiances," J. Appl. Meteorol. Climatol. 51(8), 1455-1476 (2012).

16. E. Weisz, W. L. Smith, and N. Smith, "Advances in simultaneous atmospheric profile and cloud parameter regression based retrieval from high-spectral resolution radiance measurements," J. Geophys. Res. Atmos. 118(12), 6433-6443 (2013).

17. N. R. Nalli et al., "Validation of atmospheric profile retrievals from the SNPP NOAAUnique Combined Atmospheric Processing System. Part 1: temperature and moisture," IEEE Trans. Geosci. Remote Sens. 56(1), 180-190 (2018).

18. E. Weisz et al., "Assessing hyperspectral retrieval algorithms and their products for use in direct broadcast applications," in Proc. ITSC-20 (2015). 
19. J. Le Marshall et al., "Improving global analysis and forecasting with AIRS," Bull. Am. Meteorol. Soc. 87(7), 891-895 (2006).

20. M. G. Divakarla et al., "Validation of Atmospheric Infrared Sounder temperature and water vapor retrievals with matched radiosonde measurements and forecasts," J. Geophys. Res. Atmos. 111, D09S15 (2006).

21. R. B. Esmaili et al., "Adapting satellite soundings for operational forecasting within the hazardous weather testbed," Remote Sens. 12(5), 886 (2020).

22. W. McCarty, G. Jedlovec, and T. L. Miller, "Impact of the assimilation of Atmospheric Infrared Sounder radiance measurements on short-term weather forecasts," J. Geophys. Res. Atmos. 114, D18122 (2009).

23. P. Wang et al., "The impact of Cross-track Infrared Sounder (CrIS) cloud-cleared radiances on Hurricane Joaquin (2015) and Matthew (2016) forecasts," J. Geophys. Res. Atmos. 122(24), 13,201-13,218 (2017).

24. T. J. Schmit et al., "Introducing the next-generation Advanced Baseline Imager on GOES-R,” Bull. Am. Meteorol. Soc. 86(8), 1079-1096 (2005).

25. D. Hillger et al., "First-light imagery from Suomi NPP VIIRS," Bull. Am. Meteorol. Soc. 94(7), 1019-1029 (2013).

26. M. D. King et al., "Cloud and aerosol properties, precipitable water, and profiles of temperature and water vapor from MODIS," IEEE Trans. Geosci. Remote Sens. 41(2), 442-458 (2003).

27. T. J. Schmit et al., "Many uses of the Geostationary Operational Environmental Satellite-10 sounder and imager during a high inclination state," J. Appl. Remote Sens. 3(1), 033514 (2009).

28. J. L. Bentley, "Multidimensional binary search trees used for associative searching," Commun. ACM 18(9), 509-517 (1975).

29. T. J. Schmit et al., "The GOES-R Advanced Baseline Imager and the continuation of current sounder products," J. Appl. Meteorol. Climatol. 47(10), 2696-2711 (2008).

30. M. L. Feltz et al., "Assessment of NOAA NUCAPS upper air temperature profiles using COSMIC GPS radio occultation and ARM radiosondes," J. Geophys. Res. Atmos. 122(17), 9130-9153 (2017).

31. D. C. Tobin et al., "Atmospheric Radiation Measurement site atmospheric state best estimates for Atmospheric Infrared Sounder temperature and water vapor retrieval validation," J. Geophys. Res. Atmos. 111(D9), 18 (2006).

32. "Atmospheric Radiation Measurement (ARM) user facility," 1994, updated daily. BalloonBorne Sounding System (SONDEWNPN). Mar. 2017 - Apr. 2019, 36 36' $16^{\prime \prime}$ N, $97^{\circ} 29^{\prime} 6^{\prime \prime}$ E; $36^{\circ} 36^{\prime} 18^{\prime \prime} \mathrm{N}, 97^{\circ} 29^{\prime} 6^{\prime \prime} \mathrm{E} ; 36^{\circ} 36^{\prime} 18^{\prime \prime} \mathrm{N}, 97^{\circ} 29^{\prime} 6^{\prime \prime} \mathrm{E} ; 36^{\circ} 36^{\prime} 18^{\prime \prime} \mathrm{N}, 97^{\circ} 29^{\prime} 6^{\prime \prime} \mathrm{E}$ : Southern Great Planes C1, S01, S02, S03. Compiled by R Coulter, J Prell, M Ritsche, and D Holdridge. ARM Data Center: Oak Ridge, Tennessee, USA, http://dx.doi.org/10.5439/ 1021460 (accessed 25 June 2019).

James Anheuser received his BS degree in mechanical engineering in 2012 and his MS degree in 2020 from the University of Wisconsin-Madison (UW). Currently, he is a PhD student and research assistant with the Space Science and Engineering Center (SSEC) at the UW and focuses on instrument scale relationships and remote sensing of the cryosphere.

Elisabeth Weisz received her MS degree in theoretical physics and her doctorate in geophysics and meteorology from the University of Graz, Austria. She has been with the SSEC at the UW since 2001, currently serving as an associate scientist. Her research focuses on the development of atmospheric sounding retrieval algorithm using satellite-based high spectral resolution IR radiance measurements.

W. Paul Menzel received his degree in physics from the UW in 1974. Thereafter, at the UW SSEC, he explored possibilities for sounding the Earth's atmosphere from satellites. In 1983, he joined NOAA to develop and test new remote sensing environmental products. Currently, he is a senior scientist at SSEC studying improved synergy of LEO sounders and GEO imagers and deriving global cloud and moisture records from four decades of satellite measurements. He is a fellow of the American Meteorological Society. 